\title{
Self-injury rates indicate Canadian mental health services are inadequate
}

A pproximately 45 Canadians enter hospitals each day following suicide attempts or self-inflicted injuries, a number that could be greatly reduced through improved community-based intervention strategies and greater awareness of groups most at risk for self-harm, according to the Canadian Institute for Heath Information (CIHI).

About 17482 Canadians were admitted to hospitals for overnight stays as a result of self-inflicted injuries in 2009 2010, CIHI says in a new report, Health Indicators 2011, (http://secure.cihi.ca/ci hiweb/products/health_indicators_2011 _en.pdf).

"One of the surprising things about self-injury indicators is that $70 \%$ of those admitted to hospitals have a mental health diagnosis, which means that $30 \%$ don't," says Kira Leeb, CIHI's director of health system performance. "What does that mean? Are we underdiagnosing the mental health component? The other aspect is that mental health is a continuum, and it's quite possible to self-injure without having a clinical diagnosis of mental illness."

The highest rate of hospitalization for self-injury, at 140 per 100000 people, was among women aged 15-19, with poisoning being the primary cause (85\%), followed by cutting (10\%) and strangulation (2\%). The rate for men in that category was more than $50 \%$ lower, though, overall, men were more than three times as likely to commit suicide than women (16 per $100000 \mathrm{v}$. 5 per 100000 ).

Although suicide accounts for 4000 deaths per year in Canada, placing it among the top 10 causes of death in the country, Canada lacks a national strategy for suicide prevention and efforts to create one have been mired in jurisdictional wrangling (www.cmaj.ca/lookup /doi/10.1503/cmaj.109-3724).

"The problem is breakdown in community support," says Richard Ramsay,

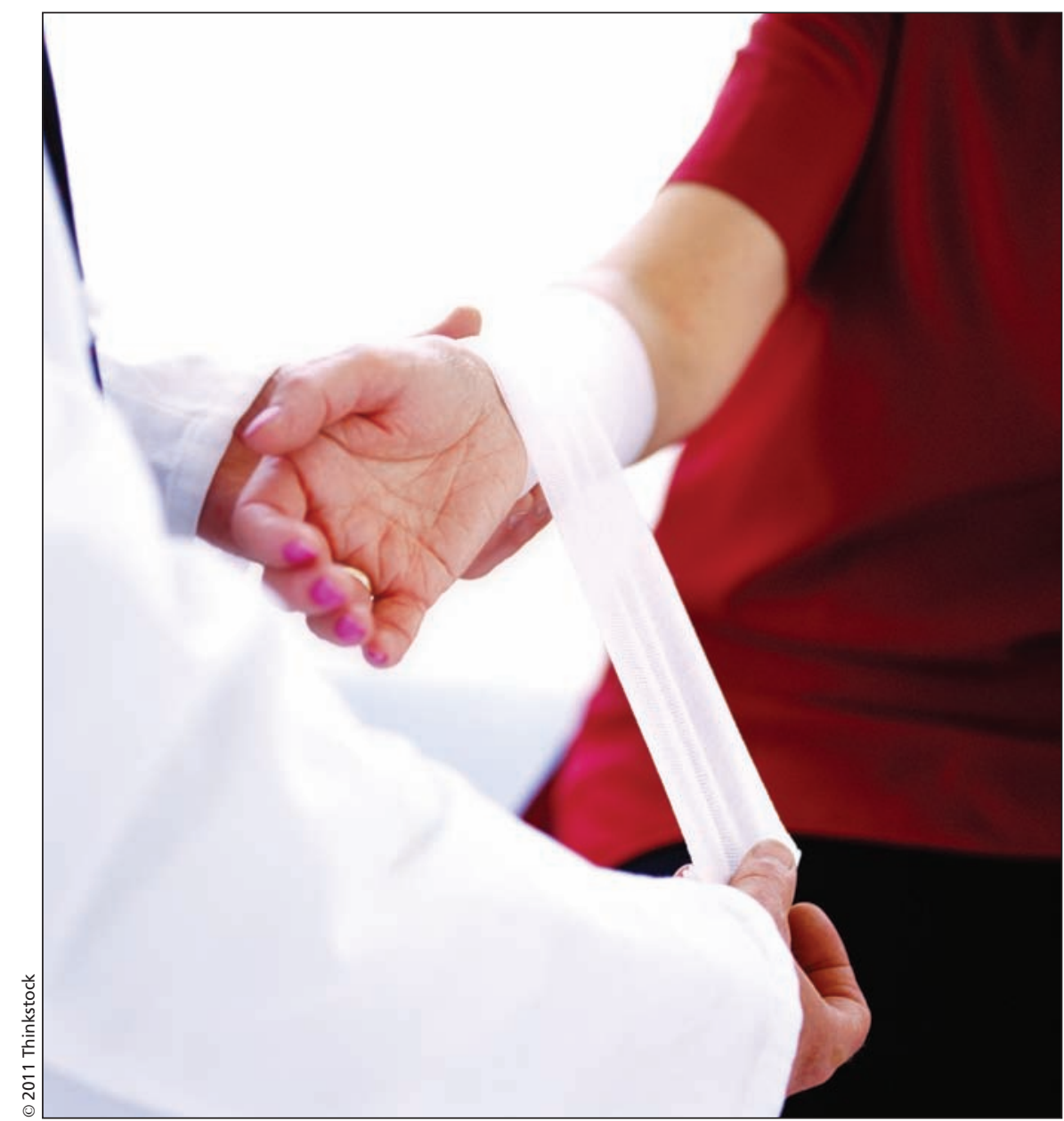

Hospitalization rates for self-inflicted injuries, including cutting, are highest among young women aged 15-19, according to a Canadian Institute for Health Information report.

president of LivingWorks, an international suicide intervention training company based in Calgary, Alberta. "We have to find ways that don't involve sending people off to a stranger for help. You need to strengthen where they came from rather than sending them to a new regime of care."

The CIHI report also indicates that rates of hospitalization due to selfinjury vary from province to province. The provinces with the lowest rates, all below 60 per 100000 , are Prince Edward Island (55 per 100 000) and Ontario and Manitoba, which both have rates of 58 per 100 000. New Brunswick, as well as Newfoundland and Labrador, have the highest rates, each at 81 per 100000 . Because of complex societal and health care issues, the rates in the territories were substantially higher than in the provinces, ranging from 192 per 100000 in the Yukon to 379 per 100000 in Nunavut.

Overall, rates of self-injury and suicide have been declining in Canada. It has been estimated, the report notes, that hospitalization due to self-injury has decreased by $16 \%$ over the past decade, and suicides have dropped by 
about $15 \%$ since 1990 . That doesn't mean, of course, that the current rates of self-harm are acceptable.

"We still have 45 Canadians a day being hospitalized for self-injury, so we still have a long ways to go," says Leeb.

Also, the actual rates of self-injury among Canadians may be as much as $50 \%$ higher than indicated by hospitalization rates, the report suggests. Some people who harm themselves don't seek medical care or, if they do, receive it in emergency rooms and aren't admitted for overnight stays. Others may die from their injuries before care can be sought.

"Despite these limitations, the rate of hospitalizations for self-injury in a general hospital may represent a partial indicator of the extent to which community-based services are accessible and effective in minimizing self-injury," the report states.

Self-injury hospitalization rates are but one measure of the access Canadians have to effective mental health services. The CIHI report also highlights two other performance indicators: 30 day mental health readmission rates and repeat hospitalizations for mental illness over a one-year period.

The 30-day readmission rate serves as a proxy measure for the coordination and continuity of mental health services. The transition from hospital to community can be a vulnerable time for patients and if community-based services are not effectively linked to hospitals, relapses are more likely to occur. Readmission rates also tend to be high when follow-up support isn't coordinated before patients are discharged and when the discharge is against medical advice. Other factors linked to readmission include a patient's age (rates decrease with age) and a history of repeat hospitalization for mental illness.

"Reducing the need for readmission by ensuring care and support are coordinated and provided following discharge is beneficial for individuals and caregivers and reduces costs," the report states.

In Canada, the 30-day readmission rate for people with mental illnesses is $11.4 \%$. As with self-injury rates, the rates of 30-day readmission vary across the country. British Columbia and Nova Scotia have the highest rates among the provinces $(12.9 \%$ and $12.2 \%$, respectively), while Manitoba (9.6\%) and Alberta (9.9\%) have the lowest. Overall, Nunavut had the lowest rate, at $7.7 \%$.
A third performance indicator, repeat hospitalizations for mental illnesses over a one-year period, is considered a proxy for a measure of the appropriateness of the mental health services available to patients. In 2008 2009, more than 10000 people were admitted to hospital multiple times in a single year for mental health care, the report states. That means $11 \%$ of people who seek in-patient care for mental health illnesses come back within a year.

"Exploring hospital utilization patterns for individuals admitted with mental illness may be useful to highlight the use and availability (or lack thereof) of outpatient/community services," it adds. "More specifically, identifying frequent users of inpatient services may help to raise questions about appropriateness of services, including where they are received, gaps in the system and opportunities for preventive efforts."

The World Health Organization says that more than one million people around the world commit suicide annually. — Roger Collier, CMAJ

CMAJ 2011. DOI:10.1503/cmaj.109-3920 